\title{
Motor-Imagery EEG-based BCls in Wheelchairs Movement and Control: A Systematic Literature Review
}

This paper was downloaded from TechRxiv (https://www.techrxiv.org).

\section{LICENSE}

$\mathrm{CCO}$

SUBMISSION DATE / POSTED DATE

06-07-2021 / 26-07-2021

\section{CITATION}

Palumbo, Arrigo; Gramigna, Vera; Calabrese, Barbara; lelpo, Nicola (2021): Motor-Imagery EEG-based BCls in Wheelchairs Movement and Control: A Systematic Literature Review. TechRxiv. Preprint. https://doi.org/10.36227/techrxiv.14916537.v2

$\mathrm{DOI}$

10.36227/techrxiv.14916537.v2 


\title{
Motor-Imagery EEG-based BCls in Wheelchairs Movement and Control: A Systematic Literature Review
}

\author{
A. Palumbo, Member, IEEE, V. Gramigna, B. Calabrese and N. lelpo
}

\begin{abstract}
The pandemic emergency of the coronavirus disease 2019 (COVID-19) shed light on the need for innovative aids, devices and assistive technologies to enable people with severe disabilities to live their daily lives. EEG-based Brain-Computer Interfaces (BCls) can lead individuals with significant health challenges to improve their independence, facilitate participation in activities, thus enhancing overall well-being and preventing impairments.

This systematic review provides state-of-the-art applications of EEG-based BCls, particularly those using motor-imagery (MI) data, to wheelchair control and movement. It presents a thorough examination of the different studies conducted since 2010, focusing on the algorithm analysis, features extraction, features selection and classification techniques used, and wheelchair components and performance evaluation.

The results provided in this paper could highlight the limitations of current biomedical instrumentations applied to people with severe disabilities in the pandemic context of Covid-19 and bring focus to innovative research topics.
\end{abstract}

Index Terms-Motor-Imagery (MI), Brain-Computer Interface (BCl), Electroencephalography (EEG), Brain Controller Wheelchair (BCW)

This work has been funded by the SIMpLE (Smart solutlons for health Monitoring and independent mobiLity for Elderly and disable people) project (CUP B69G14000180008), a Smart Cities and Communities and Social Innovation project, funded by the Italian Ministry of Research and Education (MIUR).

(Corresponding author: gramigna@unicz.it.)

A. P., B. C. and N. I. are with the Department of Medical and Surgical Sciences, "Magna Græcia" University, 88100 Catanzaro, Italy (e-mail: palumbo@unicz.it, calabreseb@unicz.it, ielpon@unicz.it).

V. G. is with the Neuroscience Research Center Magna Graecia University, 88100 Catanzaro, Italy (e-mail: gramigna@unicz.it).

\section{INTRODUCTION}

$T$ HE epidemiological context of the coronavirus disease 2019 (COVID-19) pandemic has had wide-reaching impacts on all segments and sectors of society, imposing severe restrictions on the individuals' participation in daily living activities, mobility and transport, on access to education, services and healthcare. In this scenario, the pandemic emergency shed light on the need for innovative devices, biomedical solutions and assistive technologies (AT) to facilitate persons with severe disabilities regarding their participation in daily life [1].

In recent years, the Brain-Computer Interface (BCI) application has been growing rapidly, establishing itself as an emerging technology able to translate human intentions into control signals and allow disabled people to interact with the external environment without any kinaesthetic movement [1]. It has mainly targeted patients with neurological diseases such as amyotrophic lateral sclerosis (ALS), brainstem stroke, multiple sclerosis and high spinal cord injury.

ALS is a progressive neurodegenerative disease that mainly affects motor neurones in the cerebral cortex, brainstem, and spinal cord. As the disease progresses, it led to a condition characterised by loss of ability in controlling the voluntary muscles. Subjects are aware of everything going around (the brain works properly). Still, they show limited (LIS: locked-in syndrome) or no motor response (CLIS: completely locked-in syndrome), meaning that the movement commands are not transmitted through the body limbs.

In the pandemic context, ALS patients' management has become highly complicated [2] due to suspension or postponement of the outpatient follow-up visits.

Several innovative solutions of telehealth, telemedicine [2][3] and remote monitoring systems [4] have been proposed to allow patients' follow up in remote locations using telecommunications technology.

To complement the mentioned solutions, for these people unable to easily transmit their intentions to external devices using conventional interfaces such as a mouse or a keyboard, the development of brain-controlled systems could be the optimal solution to allow them to live their daily life [5][6].

Indeed, BCI is a useful tool to establish an additional communication channel between the subjects and external devices through users' cerebral patterns. Thus, this approach 
can be efficiently used to improve their independence and facilitate participation in activities, thus enhancing overall well-being, reducing marginalisation, and preventing impairments.

Specifically, electroencephalography (EEG)-based BCI has emerged as a technology with high translational potential owing to its desirable traits: direct measures of neural activity, portability, non-invasiveness and inexpensiveness [7].

EEG-based BCI technologies in controlling mobile robots, particularly wheelchair systems, have been the subject of recent research interest.

Several contributions have been published during the last decade to provide state-of-the-art wheelchairs driven by a brain-computer interface. Two of them [8][9] contain a survey, partially connected to this field, on brain-controlled mobile robots, describing the overall systems and the key techniques and the evaluation parameters of these robots. The other three articles [10][11][12] presented an extensive overview of current BCI-based wheelchair solutions. A recent paper [13] provides a detailed review of EEG signal processing in robot control (mobile robots and robotic arms), mainly based on non-invasive brain-computer interface systems.

Our paper aims to present the state-of-the-art applications of EEG-based BCIs, particularly those using motor-imagery (MI) data, to wheelchair movement and control in a real environment.

Focusing on the applicability and feasibility of braincontrolled wheelchair in the pandemic context and highlighting the need for easy usability required for disabled people, we considered studies that are based only on motor imagery EEG data and that tested the BCI approach on a real wheelchair, or at least a prototype, but not a simulator.

The review presents a thorough examination of the different studies conducted since 2010, focusing on the algorithm analysis, features extraction, features selection and classification techniques used, and wheelchair components and performance evaluation.

The rest of this paper is organised as follows: Section 2 describes more in details the methodology used for this review. Section 3 presents a synthetic overview of BCIs classifications and applications, identifying our area of interest more precisely.

Section 4 focuses on applying MI EEG-bases BCIs in wheelchairs movement and control and summarises the different existing solutions.

Section 5 discusses algorithm analysis, features extraction, features selection, classification techniques and software used in the selected contributions. Finally, Section 6 presents the main conclusions of this study and focuses on the primary challenges of biomedical research applied to people with severe disabilities in the pandemic context of Covid-19.

\section{Methodology FOR this REVIEW}

\section{A. Search Strategy}

This systematic review was conducted following the preferred reporting items for systematic reviews and metaanalyses [14].
A comprehensive literature search was conducted on March 1, 2021. The most common engineering and medical databases (IEEE Xplore, Pubmed, Science Direct and Scopus) were selected for research.

The review was limited to texts published in English between 2010 and 2021, for which abstracts were available.

Considering the scope of the systematic review, the specific keywords were defined. This structured search string was used to organise this paper: "motor imagery" - AND - "EEG-based" OR "electroencephalography-based" - AND - "BCI" OR "Brain-Computer Interface" - AND - "Wheelchair movement" - AND - "control".

To increase the likelihood that all the relevant studies were identified, additional articles identified through the reference list of previously retrieved articles were included.

\section{B. Inclusion and exclusion criteria}

Articles were considered for inclusion only if: (1) they described brain-computer interface systems based on motor imagery paradigms as mainly EEG acquisition modality; (2) they partially or totally demonstrate the feasibility, effectiveness and applicability of MI EEG-based BCIs for wheelchair movement or control in real-world settings; (3) they described completed research.

The articles were also screened for the following exclusion criteria: (1) contributions that described BCI systems mainly based on face gestures or intentional blinks to control wheelchair; (2) studies that described only a simulated system or virtual environment and in which there is no reference to real wheelchair (or prototype) movement and control; (3) studies that presented a hybrid-mental approach: wheelchairs that are based on more than one type of EEG signal (e.g. ERD/ERS and P300).

Exclusion criteria were also related to papers, books or book chapters, letters, review articles, editorials, and short communications.

\section{Study selection}

Our work aims to present state-of-the-art applications of EEG-based BCIs using motor-imagery data to wheelchair movement and control. Since these interfaces' target population are older people or patients with impaired motor abilities and considered our interest in assessing applicability in a daily context, only the studies that described a real wheelchair, or at least a prototype, but not a simulated system, are investigated and reported.

A particular emphasis was given to studies that performed experiment evaluation of wheelchair navigation in a realworld environment.

A total of 134 search results were identified through database searching and additional sources. After removing all duplicates, 117 studies underwent title, and abstract screening and the inclusion criteria were examined. The full texts of 22 papers assessed for eligibility were carefully analysed. Three articles [15][16][17] were excluded for exclusion criteria (1), one contribution [18] for exclusion criteria (2) and two scientific results [19][20] for exclusion criteria (3).

Finally, only 16 studies were included in the quantitative synthesis. The methodological approach is presented in Fig. 1. 
For facilitating analysis and comparisons, we summarised all relevant BCI existing solutions and related system parameters in Table I. In Section 4, we discussed each study, contextualising the results in the BCI realm.

\section{BRAIN-COMPUTER INTERFACES CLASSIFICATIONS AND APPLICATIONS: A SYNTHETIC OVERVIEW}

Far from representing an exhaustive and detailed description of BCI systems' main characteristics and classifications, discussed in depth in several contributions [21][22], this section aims to frame the area of interest of our review more precisely.

According to their invasiveness, BCIs can be classified into invasive and non-invasive ones, depending on whether sensors used to measure brain activity penetrate the skin or not [23]. In invasive BCIs, cerebral signals are acquired inside the brain using electrodes located under the skull. The two invasive modalities mainly used in BCI research are intracortical recording and electrocorticography (ECoG).

We focused our research on the non-invasive BCIs in which brain signals are acquired using sensors placed on the scalp.

Among various non-invasive brain-imaging methods often used to implement BCI systems (EEG, Magnetoencephalography (MEG), Positron emission tomography (PET), functional magnetic resonance imaging (fMRI), and Functional Near-Infrared Spectroscopy (fNIRS)), over the last couple of decades, EEG has been the most widely employed due to its desirable traits, namely non-invasiveness, portability, high temporal resolution, and a relatively low cost compared to other neuroimaging methodologies [21][24].

In recent years, up-and-coming practical applications of EEG-based BCI with several elaborately designed paradigms [25] are being evaluated [26]-[31]

Within EEG-based BCI paradigms, two groups can be roughly identified: exogenous (or evoked), which use external triggers (flickering LEDs or auditory beeps) to evoke discriminative brain patterns, and endogenous (or spontaneous), which use self-regulation of brainwaves without external stimuli [24].

Typical examples of exogenous BCI paradigms are the steady-state visual evoked potential (SSVEP)[26][27] and the P300 signal [28][29].

P300 [32][33] is a localised brain pattern response to an external attended visual, auditory, or tactile stimulus and is mainly measured in the parietal lobe.

SSVEP [34][35] is a response to a visual stimulus at a frequency greater than $6 \mathrm{~Hz}$, which can be primarily observed in the occipital area.

On the other hand, Event-Related Desynchronization/Synchronization (ERD/ERS) changes, elicited during the performance of mental tasks (e.g., motor imagery, mental arithmetic, and mental rotation) [30][31], are representative of endogenous BCIs paradigms, as they do not use any external stimuli.

Since controlling a wheelchair requires that the visual channel remains dedicated to the maintenance of visual attention on the environment, the endogenous signals, particularly those elicited by MI, are to be preferred.

Although learning to modulate endogenous signals requires more time for the users, MI paradigms present significant advantages that should not be overlooked for this review's scope.

Indeed, they do not require any external stimulation. They can be operated via free self-control and, consequently, they are particularly suitable and advantageous for the patients suffering from motor neuron diseases [24].

In light of these observations, EEG-based BCIs, particularly those using motor image paradigms applied to wheelchair apparatus control, represent the sub-area of interest of our review (Fig. 2).

\section{MI EEG-BASED BCIS IN WHEELCHAIRS MOVEMENT AND CONTROL: LITERATURE RESULTS}

In the context of the COVID-19 pandemic emergency, among various $\mathrm{BCI}$ applications, using the human brain in wheelchairs movement and control is attracting widespread attention in the scientific community due to its flexibility and potential to help old and paralysed individuals gain independence and potentially improve their quality of life.

Since the first demonstrations of feasibility that the "human mind can control a wheelchair" [36][37], several protocols have been proposed, and a sophisticated algorithm has been implemented to extend the applications of EEG-based BCIs to wheelchair movement and control [11].

Following the definition given in [13], the wheelchair is classified as a mobile robot that can navigate two dimensions.

EEG-based wheelchair system refers to a type of braincomputer interfaces technology in which this specific mobile robot is controlled using electroencephalographic patterns collected from the human brain. This technological approach allows the subject to reach a particular target using only brain signals.

Despite the enormous interest in implementing a braincontrolled wheelchair (BCW) that can improve disabled people autonomy allowing them to move through a real environment [10], the number of scientific contributions in the field is not very high due to the complexity of developing such elaborate system [38].

In presenting the background of recent studies on wheelchair control through the acquisition of a user's brain activity, the groups of Al-qaysi [11] and Fernández-Rodríguez [10] analyzed several BCW existing solutions: MI-based BCW [39]-[41], P300-based BCW [42]-[46], SSVEP-based BCW [47]-[51] and hybrid-based BCW [52][53]. Hybridbased $\mathrm{BCW}$ is commonly identified as a system based on one EEG input combined with one or more channels (e.g. EEG, electromyography (EMG), electrooculography (EOG) or movement detection).

All these studies present a standard signal acquisition methodology (EEG) to control the system, but different structural elements: the specific signals used to implement the BCI system, the tasks to be performed by users, the number and type of commands available on the device, the modality of navigation, etc.

As mentioned above, our interest is to prove the feasibility and applicability of a brain-controlled wheelchair in a real environment considering, as the target population, patients with impaired motor abilities. 
For this reason, among the four EEG control signals models used to handle BCI wheelchairs, those based on motor-imagery task can be considered the most appropriate choice for achieving the intended purpose. Indeed, a motorimagery paradigm does not rely on visual stimuli and does not interfere with navigation's visual task, allowing the user to control the wheelchair spontaneously. The subject is not exposed to any stimulation, and thus there is no risk of fatigue.

In addition, a brain-controlled wheelchair based on the motor imagery paradigm is more appropriate for use in an unknown environment, and several classes of identified motor imagery output can be directly transmitted into the directional control of a robotic wheelchair [54].

Finally, the use of MI when dealing with motor-disabled patients makes sense since this paradigm does not interfere with the patient's residual capabilities, involving a part of the cortex that may have effectively become redundant [55].

This systematic review focuses on key issues related to noninvasive EEG-based BCIs that use motor imagery as the main paradigm applied to wheelchair movement and control.

In this section, several existing applications of MI EEGbased BCIs for wheelchair movement and control are illustrated. These studies are carefully analysed, and the main characteristics in terms of signal acquisition, preprocessing, feature extraction and classifications methods, and wheelchair performances evaluation were summarised in Table I. The studies are tabulated in chronological order.

Tsui et al. [54] presented a simple 2-class self-paced MIbased BCI for wheelchair control. With this system, the user was able to make path planning and fully control the wheelchair. Based on a laser range finder, an automatic obstacle avoidance system is integrated with the robotic wheelchair's control mechanism.

The work of Carrino et al. [56] proposed a user-friendly, self-paced BCI system that, using a commercial EEG headset and a motor imagery approach, allows the user to drive an electric wheelchair. Although the low-cost EEG device provided interesting results, the authors stated that it could hardly be used for self-paced systems in error sensitive context. Indeed, the system was tested directly on the wheelchair, and several problems occurred. More specifically, the classification process's errors produced an unexpected behaviour of the wheelchair and, thus, a strong perturbation for the user, concentrated in motor imagery tasks. Since this problem does not allow any kind of navigation for non-trained subjects, the test was finally performed using real gestures, less sensitive to emotional perturbations.

A novel wheelchair system controlled by EEG signals was constructed by Choi et al. [57], using effective signal processing methods to allow people paralysed from the neck down to interact with society more freely. The authors confirmed that the proposed wheelchair system demonstrated almost the same performance as a wheelchair controlled by a joystick.

In $\mathrm{Li}$ et al. [39], authors evaluated the feasibility of BCIbased wheelchair, which users' thoughts can steer without any additional involvements. In practical driving testing in a real environment, the system achieved a good performance, suggesting a potential application to people with disabilities in daily life.

The group of Carra [58] illustrated the development of a non-invasive experimental BCI system. The proposed approach generated commands to move a motorised wheelchair using portable and low-cost equipment and capturing the brain signals from the somatosensory cortex without the involvement of peripheral nerves and muscles. Experimental tests performed in an uncontrolled environment showed promising results, thus enabling a possible future interface with real-life situations.

For designing a BCI system, Reshmi et al. [59] introduced five-class motor imagery EEG-based approach. The patterns acquired from the sensory-motor cortex are translated into a control signal to manage the directional movement of a wheelchair. Indeed, users' movement intentions are classified according to the limb movements, and the results of patterns identification can be used as a command to move and stop the wheelchair.

Carlson et al. [55] proposed an asynchronous wheelchair system, integrated with robotics and computer vision techniques, that allows the subject to control the wheelchair by performing a motor-imagery task spontaneously. This group introduced the notion of shared control to integrate the user's intelligence with the precise capabilities of a robotic wheelchair given the context of the surroundings. The authors demonstrated that several types of BCI wheelchair operators (new and experienced ones) could complete a navigation task successfully. Moreover, compared with an alternative P300based system, the asynchronous MI approach gives users greater flexibility and authority over the actual trajectories driven. More specifically, the users can interact with the wheelchair spontaneously and can voluntary control the motion at all times, rather than having to wait for external cues. Besides, they can dynamically produce intuitive and smooth trajectories rather than relying on predefined routes, thus reducing the inactivity navigation time.

To overcome some of the limitations of several existing solutions, such as gaze dependence and unnecessary stops, Kim et al. [60] presented an MI-based brain-actuated wheelchair system using an extended five command protocol. The presented wheelchair could be driven by the user in both smooth and right-angled turns. The user could be integrated with various robotic and computer vision sensors via additional channels in the network module for safety improvement. This approach can allow the user to cope with various environments, reaching a goal point with lower execution time.

In the study of Varona-Moya et al. [40], the authors tested the feasibility of driving a customised robotic wheelchair with an MI-based BCI system and the auditory cues to inform the subject of the available navigation command at every moment. To enable effective and autonomous wheelchair navigation, this group proposed an application interface that, based on a two-class sensorimotor rhythms-BCI paradigm, provided the user with four navigation commands. The results suggested 
that this system seems to be an effective way of driving a robotic wheelchair autonomously and could provide locked-in patients with a better quality of life.

Swee et al. [61] proposed developing an electric wheelchair that can be directly controlled by the brain and that does not require any physical feedback as controlling input from the user. The authors anticipated that this system could give a new contribution to physically disabled people to regain their mobility.

Zhang et al. [62] demonstrated the effectiveness of a braincontrolled intelligent wheelchair that combines an MI- (or P300-) based BCI and an automated navigation system. For the scope of this review, only the MI-based BCI solution was taken into account. The proposed wheelchair has several advantages: (i) it can adapt to changes in the environment; (ii) once the user selects a destination with the BCI, the system automatically navigates to it, allowing the workload reduction for the user; (iii) during the wheelchair navigation, the user can issue a stop command via the BCI.

To provide several navigation commands without worsening the system performance, a paradigm based on the discrimination of only two mental tasks to control the wheelchair is presented in the study of Ron-Angevin et al. [38]. Such a non-muscular control system has the peculiarity that it is embedded with an auditory interface that provides the user with four navigation commands. The authors suggested that this system could be an effective option to allow wheelchair displacement in a controlled environment for potential users with motor neuron diseases in the face of more extensive training.

The group of Al-Turabi [63] described the experience of developing a complete BCI system able to instruct a wheelchair to move to different directions using non-invasive EEG brain waves. In light of the experimental results, the authors proposed their system to control other devices and hypothesised, as future improvement, a cloud-based system to direct communication from the headset to the wheelchair.

In $\mathrm{Yu}$ et al. [64], the authors implemented an asynchronous control strategy in which the wheelchair commands are generated by a multi-step process based on sequential MI, without any external prompt information. Although the system was tested in healthy subjects, the preliminary experimental results demonstrated this navigation strategy's potential applicability in enhancing the mobility of people with physical disabilities in a real environment.

Permana's project purpose [65] was to control the wheelchair using a motor imagination-based BCI and a portable EEG device. In performing a preliminary experimental test using only MI patterns from a single data channel to trigger the wheelchair movement, the authors found some problems. Indeed, due to similarities in EEG patterns related to different motor paradigms, the classification for several wheelchair control signals failed. To overcome this limitation, they added a new variable (eyes motion) as a differentiator of similar data without obtaining evaluable results. In conclusions, our method needs to be developed and improved.
In a recent work of Xiong et al. [7], authors have made several important contributions to the state-of-the-art in BCIs. They proposed a wheelchair prototype that uses hand motor imagery and jaw clench data collected with a consumer-grade EEG system, bridging the gap between the real-time classification of motor imagery and the use of a low-cost apparatus. Although future experiments and a consistent neurofeedback training procedure are required to validate their prototype, this system seems to get closer to the actual context's needs and demands. Authors highlighted that this system's clinical applications would largely depend on the motor abilities of the user (EMG toggle would be inaccessible to patients with more severe disabilities such as CLIS) and proposed, as future work, other integrative non-muscular signals, such as electrooculography (EOG).

\section{MI-BASED BCW ELEMENTS}

In the design and implementation of a brain-based control wheelchair system, four stages are necessary to establish the communication between the human brain and the external device and to get a useful output to be used in controlling it: brain signals acquisition, preprocessing, features extraction from patterns and features classification. An example of braincontrolled wheelchair components and the system application in a real environment is illustrated in Fig. 3 .

A successful BCI system must be characterised by the best accuracy in extracting EEG features and classifying them. Indeed, since the presence of errors can cause the initiation of a wrong command that can lead to dangerous situations, a high classification rate and accuracy are required [17]. For this reason, features extraction and classification processes play a significant role.

In this section, we discussed these four stages in more detail, together with published examples and in the light of summarised results. As integration, the software libraries primarily used in the collected studies were illustrated and analysed.

\section{A. Signal acquisition}

As mentioned above, this systematic review focuses on the non-invasive methods on which the applications of EEG-based brain-controlled wheelchair are based.

Many EEG data acquisition devices are available in commerce, which vary in the number of channels, sampling rate, electrode connection type, headset preparation time, and price [13][66][67].

Our review results revealed that, in MI-based braincontrolled wheelchair applications, the most used devices to capture EEG signals are Epoc [68], produced by Emotiv Systems Inc., and the g.tec medical engineering products, such as gUSBamp.

The Emotiv Epoc is a portable, high-resolution EEG system with 14 dry electrodes designed to be quick and easy to fit, taking practical applications measurements. Many BCI studies used EPOC to control or interact with machines in users' environment [69], although the validity of Emotiv products in 
clinical research is still a matter of debate.

On the other hand, g.USBamp RESEARCH [70], a highperformance and high-accuracy 16 channels biosignal amplifier, was proposed by the g.tec medical engineering to acquire and process physiological signals.

g.USBamp has become a widely used standard for neurophysiological research, life sciences, neurofeedback and brain-computer interface approach.

Also, Brain Products EEG amplifiers [71], such as BrainAmp DC [64] and Acti-CHamp [40], were commonly used for a variety of practical uses in neurophysiological research.

An overview of other used EEG signal recording devices is presented in Table I, together with examples of associated brain-controlled wheelchairs.

\section{B. Pre-processing}

Signal preprocessing is a non-trivial step required to clean data and remove any unwanted components (noise, artefacts or interference) embedded within the EEG signals [13][21]. A proper preprocessing procedure produces an improvement in the signal quality and results in better features separability and classification performance.

The most common methods applied in BCIs preprocessing and adopted in the summarised scientific contributions are frequency-domain filtering and spatial filtering. Bandpass filters [7][38][40][58][59][62][64], the primary attempts to attenuate artefacts in the measured EEG, and notch filters [57][64], used to remove the noise generated by the power line, are examples of frequency domain preprocessing solutions. However, only when the frequency bands of the signal do not overlap, these methods are effective.

A spatial filter is an alternative approach to increase the signal-to-noise ratio (SNR) of the brain signal. Typical examples of spatial filtering methods are Laplacian filtering [55][56], blind source separation (BSS)[57], common average reference (CAR) [39][60], autocorrelation (AC), canonical correlation analysis (CCA), independent component analysis (ICA), minimum energy combination (MEC), and principal component analysis (PCA). For real-time BCI applications, automatic methods and low computational cost are required. Recently emerging algorithms, such as independent vector analysis (IVA), a modified joint BSS approach (JBSS), a quadrature regression IVA (q-IVA) and the filter-bank-based supervised machine learning approach, introduced more effective artefact removal approaches, paving the way for innovative and helpful research in BCI field [72]. A detailed description of the mentioned methods out of this review's scope can be found in [13][21][22][73][74].

\section{Feature extraction}

Although the feature extraction methods used in the selected studies are quite heterogeneous, analysis in the spatial domain using Common Spectral Patterns (CSP) resulted in being the general approach [39][57][60][62][64].
Started to be employed since 2000 to detect event-related desynchronizations [75], CSP filter is mentioned as an effective way to discriminate classes and one of the most popular feature extraction methods in the BCI field [22]. This method aims to transform EEG data into a new space, maximising the variance of the (projected) signal from one class and minimise it for the other class.

It is considered a strong technique in MI EEG processing since it enables the extraction of signal information from particular frequency bands.

However, proper selection of the filtering frequency band dramatically affects the performance of CSP, and the optimal frequency band is typically subject-specific. Thus it is difficult to select manually.

The common spatial frequency subspace decomposition (CSFSD) method, adopted in Choi et al. [57], is a modified type of CSP [75].

In other summarised BCW systems, the power spectral density (PSD) method is also adopted. PSD is the measure of how the power of a signal is distributed over frequency.

It is an efficient frequency domain based feature widely used in motor imagery paradigms, but its performance may decrease seriously when applied to low SNR data.

\section{Pattern classification}

Implementing a successful BCI approach requires that the system identifies several user brain activity patterns, extract from them the most significant features, and classify them with the best accuracy. The classification step compares the user's intention into command signals for an output device (for example, a wheelchair).

Especially in third-party device control applications, where errors can lead to dangerous situations, high classification rate and accuracy are mandatory [17]. Although either regression or classification algorithms could be employed to achieve the goal above, the latter's use is considered to be the most popular approach [76]. Among the numerous classification algorithms commonly used in BCI's scientific context [21] and especially in brain-controlled mobile robots [13], the SVMbased and LDA-based approaches predominate in the studies of this review.

LDA is an effective statistical technique used as a wellknown binary classification method for EEG data. It is employed to identify the linear combinations of feature vectors that characterise the corresponding signal. This method projects data in a new space and uses a hyperplane to distinguish different classes, minimizing the variance within a class and maximising the variance between classes [13].

Thanks to its satisfactory performance, very low computational cost, easy use and no requirement of extensive pretraining, an LDA classifier is to be preferred in various BCI systems, for example, in motor imagery-based approach. However, its linearity can cause performance degradation and poor results in a few circumstances containing complex large non-linear EEG data.

On the other hand, SVM, first proposed by Vapnik et al. [77], is a supervised learning algorithm used to solve binary 
classification problems by creating a linear optimal hyperplane. To perform a classification process for a given set of training data, SVM constructs a hyperplane model in a multidimensional space that separates the patterns belonging to the different classes by the widest margin [13]. As reported in Padfield et al. [22], the SVM classifier resulted in higher performance when compared to LDA and regression algorithms.

In addition to LDA and SVM classification solutions, artificial neural network (ANN) and k-nearest neighbour (k$\mathrm{NN})$ [78] are also adopted.

The element that identifies the neural network $(\mathrm{NN})$ is identifying trends challenging to find, either by humans or by computerised techniques. An ANN is a multi-class classifier, widely used in the BCI field, based on a brain-inspired information system that simulates and replicate the process of human cognition [13].

$\mathrm{KNN}$ is a supervised learning algorithm that can be used to classify between two or more patterns. It is based on the concept that features related to different patterns will results in different clusters in the features space, while similar patterns will form convergent or alike clusters. The BCI community doesn't seem to widely use this method due to its sensitivity to the curse-of-dimensionality, which causes it to fail in several experiments.

\section{E. Software platforms}

Several commercial software, toolbox and framework were adopted to implement the necessary steps for EEG signals processing, such as filtering, artefact correction, feature extraction, and classification.

An overview of the most widely-used BCI platforms is presented, together with examples of associated braincontrolled wheelchairs (Table I). Besides, several features that could be essential in wheelchair control application are highlighted for each of them.

OpenVibe [79], developed by the French National Institute for Research in Computer Science and Control (INRIA), is a free and open-source platform to design, test and use BCI systems in both real and virtual environments. Adopted in Carrino et al. [56], it can be used for real-time processing and analysis of brain signals (acquire, filter, process, classify and visualize data) due to its modularity features, portability and flexibility.

OpenBCI GUI [80], used in Xiong et al. [7], is an OpenBCI's software that offers a powerful tool for visualizing, recording, and streaming data the OpenBCI Boards. Data can be transmitted in live-time to third-party software such as MATLAB.

Matlab is also a powerful tool for researchers to test models and algorithms in the BCI field, benefiting from resourceful toolboxes and easy implementation process [81]. It was adopted in most of the summarised studies.

LabVIEW (National Instruments, Inc.), a high-level multiplatform graphical development environment, was also used to implement a brain-controlled wheelchair [58].

Although not used in any of the studies summarised in this review, it is worth mentioning, within the brain-controlled wheelchair research, other open-source BCI platforms: Wyrm,
BCI2000, BCILAB and Gumpy.

Wyrm [82][83] is an open-source Python-based BCI package applicable to a broad range of neuroscientific problems. The toolbox offers several functionalities to analyse and visualise neurophysiological data in offline processing and real-time settings, like an online $\mathrm{BCI}$ application.

BCI2000 [84][85] is an open-source C++ based software developed in 2000 for real-time BCI systems application. It includes stimulus presentation functionality and provides the data acquisition and signal processing modules. Still, some important methods (e.g. discrete wavelet transform) and some classification techniques (e.g. deep learning) are not embedded. Since this software does not directly support other programming languages such as Matlab or Python, it is not easy to extend or integrate it with other toolboxes [86].

BCILAB [87][88], developed by "Swartz Center for Computational Neuroscience" (SCCN) and distributed free to help researchers in processing signal with Matlab, is among the earliest publicly available software packages for research purposes in the BCI community. The toolbox supports several feature extraction methods, experimental paradigms and classification approaches (LDA, Quadratic Discriminant Analysis (QDA) and SVM).

Gumpy [86][89] provides state-of-the-art algorithms, signal processing methods and classification approaches that the scientific community has employed over the last 20 years. Gumpy is designed for a hybrid brain-computer interface and is implemented to chart a route ahead for new BCI improvements [86]. It is an open-source Python toolbox suitable for EEG and EMG bio signal analysis, visualisation, real-time streaming and decoding. More importantly, in addition to classical machine learning algorithms, Gumpy includes different deep learning models such as deep convolutional neural networks (CNN)[90], recurrent convolutional neural networks (RCNN) and Long Short-Term Memory (LSTM)[91] which can be used to classify sensorymotor rhythms from EEG signals.

\section{CONCLUSIONS}

Our scientific research group's interest has been focused for years on the development of innovative technological solutions and assistive systems designed to preserve communication and interaction with the external world in people with ALS [4][92]-[98].

The pandemic emergency of COVID-19 has shed light on the needs of people with severe disabilities regarding their participation in daily living activities, mobility and transport, on access to education, services and healthcare.

Therefore, in this context, it was necessary to highlight the limitations of current biomedical instrumentations applied to people with severe disabilities to pave the way for future and helpful research in the BCI field.

This systematic literature review aims to prove the feasibility and applicability of a brain-controlled wheelchair in a real environment considering, as the target population, patients with impaired motor abilities.

Wheelchairs are among the most appropriate equipment that can promote mobility and improve people autonomy, 
providing valuable aid, particularly to the elderly and people with physical impairments, to move through a real environment and to do daily routines and tasks with ease.

In implementing an efficient brain-controlled wheelchair, three main challenges need to be considered: (i) system control is multi-objective, including numerous and different commands (start and stop, direction and speed). The system based on a navigation approach with a specific mental task can offer only a few navigation commands. On the other hand, an high number of cognitive tasks increases the number of navigations commands, but it can worsen the BCI system's performance. Producing numerous control signals is challenging for an EEG-based BCI, and making an accurate control command is a time-consuming process. (ii) The efficacy and the performance of a BCI system largely depend on the user who often fails to perform the MI required to produce direction control signals. (iii) Continuous control of wheelchair navigation may produce a large mental workload for the user, especially for disabled people.

Despite the enormous interest in implementing a braincontrolled wheelchair, the current solutions do not seem to fully satisfy the demands in today's pandemic context, mainly due to the complexity of developing such an elaborate system. Although innovative technological solutions of considerable importance have been implemented in MI-BCI research, some critical issues still need to be resolved [74].

Firstly, as most of the published studies are based on synchronised MI-BCI in offline modality, there is a need to give more prominence to online BCI studies.

In addition, the performance of a designed brain-controlled wheelchair should be quantified while navigating a predefined set of common obstacles.

Several performance evaluation metrics were used by researchers, as per their convenience. However, researchers have no standard performance metrics that could be widely adhered to facilitate comparisons between brain-computer systems. Improving the performance of $\mathrm{BCW}$ is still a critical issue even after two decades of research. Taking advantage of sophisticated algorithms availability, future research in MIBCI should concentrate more on reducing long calibration, on increasing the number of commands without, however, producing a large mental workload for the user. BCI illiteracy, reported in [74] as the users' inability to produce required oscillatory pattern during motor imagery paradigm, leads to poor performance of MI-BCI. The current trend of researchers is to predict whether a user falls under BCI illiterate category or not and to use this information to improve the implementation of an optimal algorithm for decoding MI and design a better training protocol for enhancing user skills.

In conclusion, we hope the results provided in this paper will highlight the limitations of current biomedical instrumentations applied to people with severe disabilities in the pandemic context of Covid-19 and bring focus to innovative research topics.

\section{ACKNOWLEDGEMENT}

This work has been funded by the SIMpLE (Smart solutIons for health Monitoring and independent mobiLity for Elderly and disable people) project (CUP B69G14000180008), a Smart Cities and Communities and Social Innovation project, funded by the Italian Ministry of Research and Education (MIUR). 


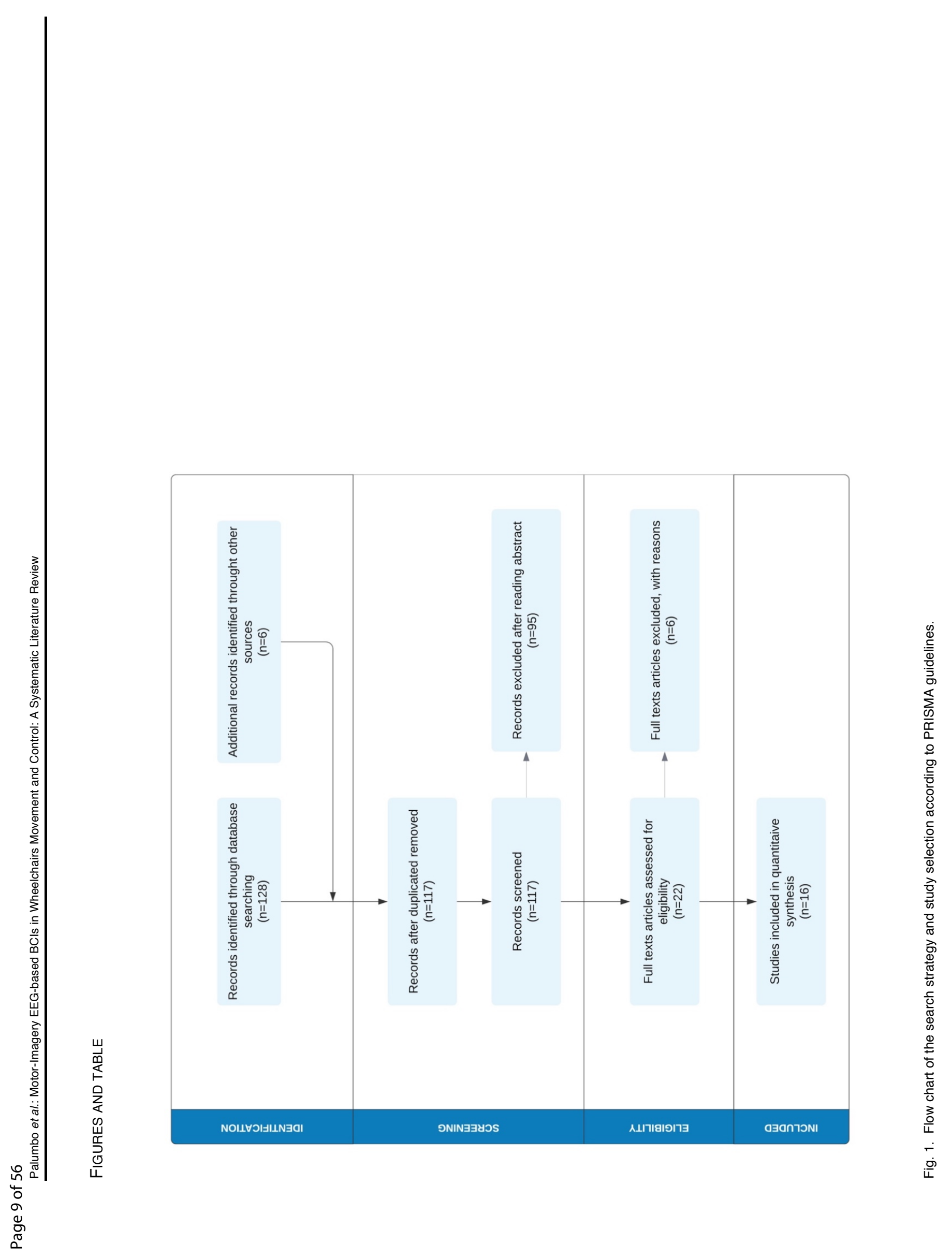




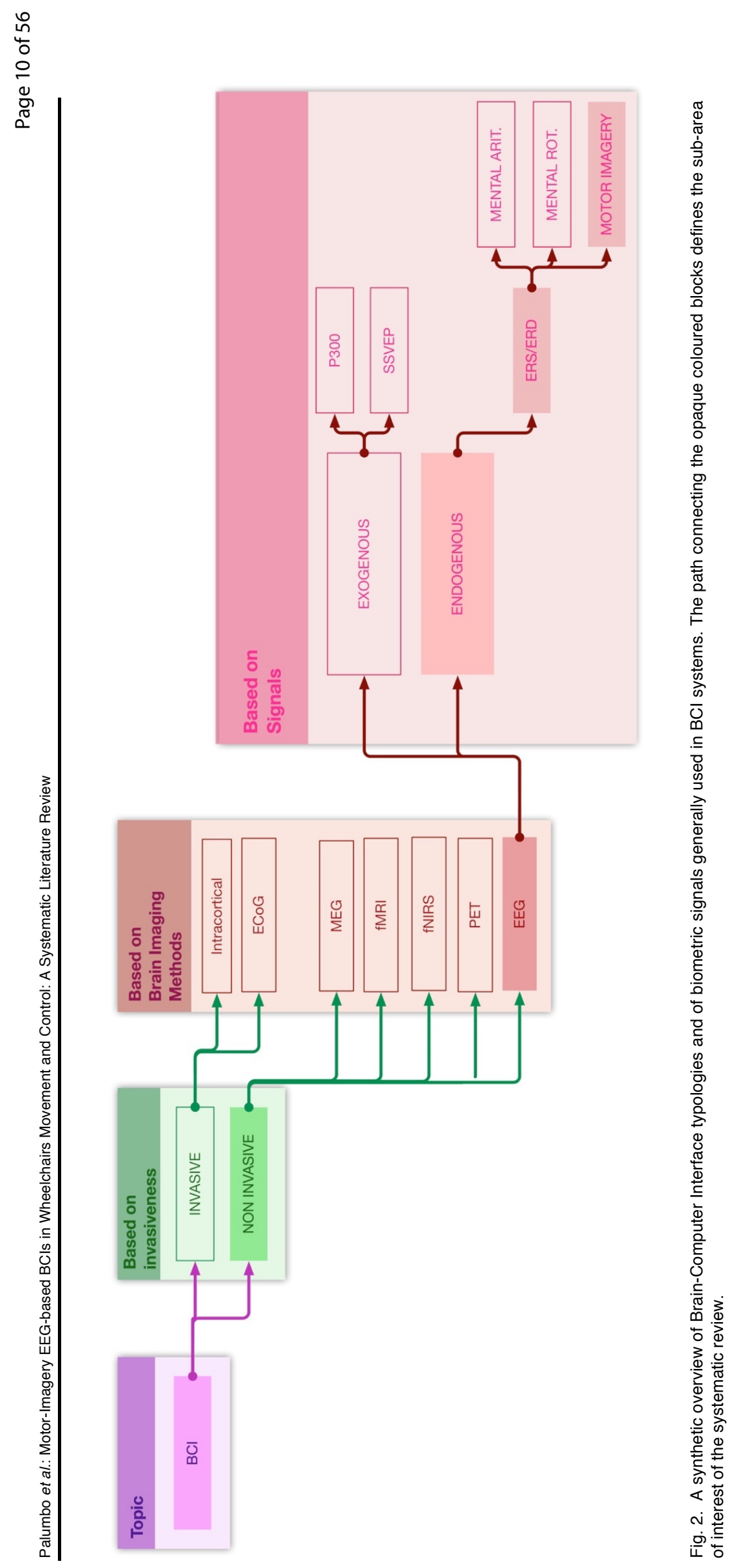




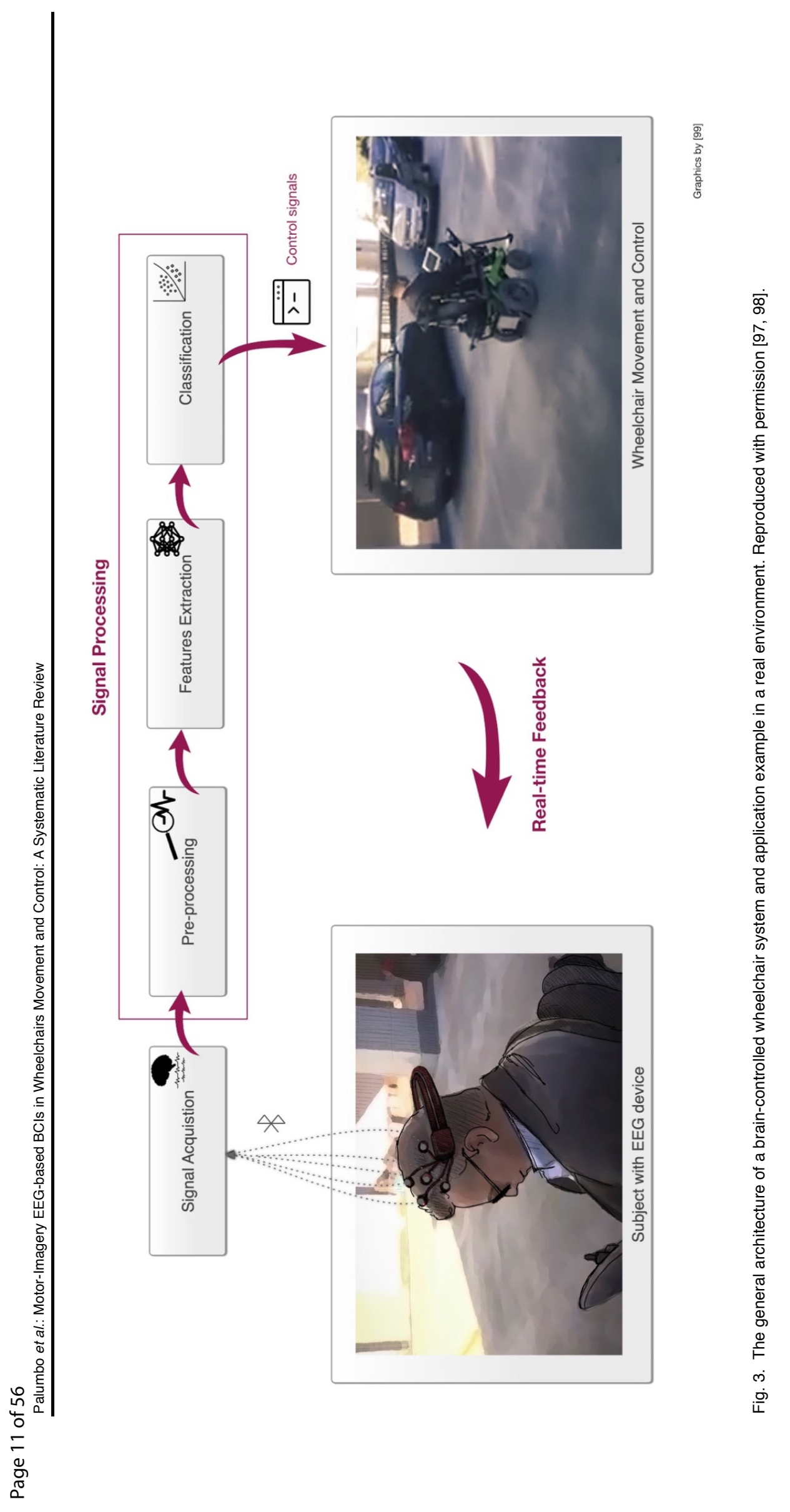




\begin{tabular}{|c|c|c|c|}
\hline 竞 & 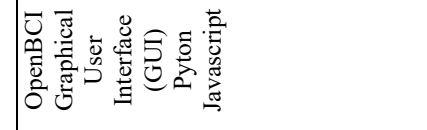 & $\frac{\substack{\frac{\pi}{\pi} \\
\frac{\pi}{\Sigma}}}{2}$ & $\ddot{\ddot{I}}$ \\
\hline 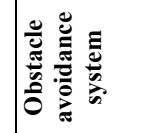 & 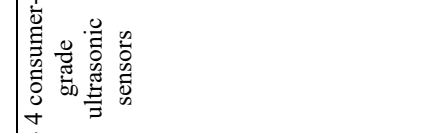 & そ & そ \\
\hline 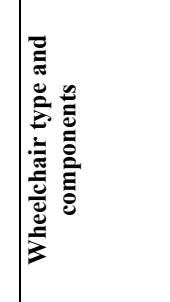 & 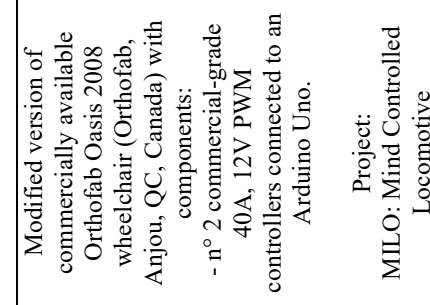 & 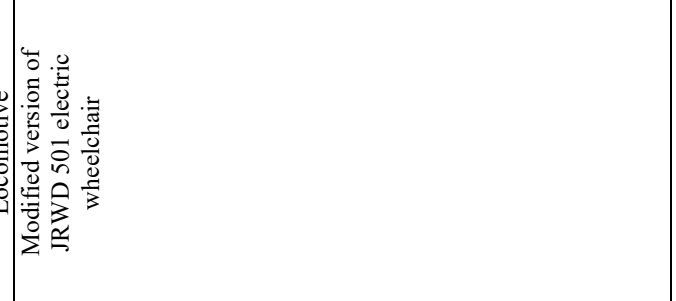 & 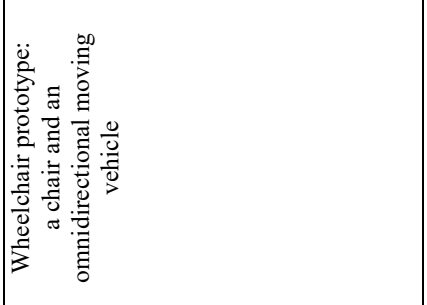 \\
\hline 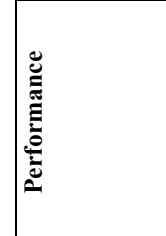 & 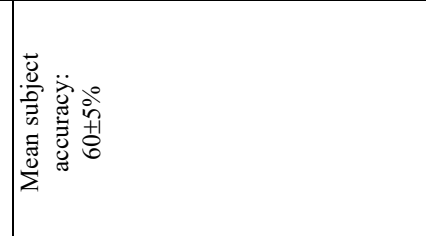 & 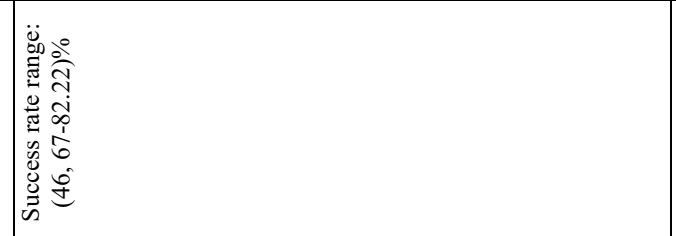 & 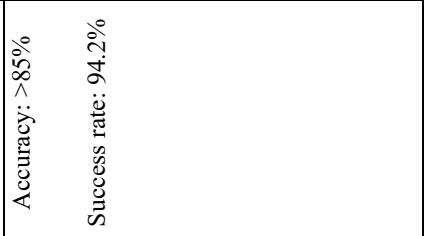 \\
\hline 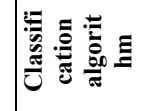 & 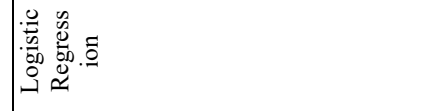 & 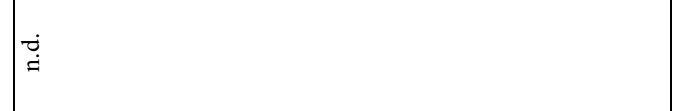 & 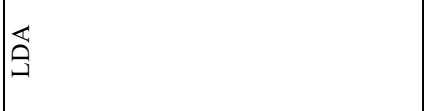 \\
\hline 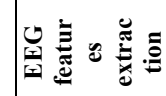 & की & 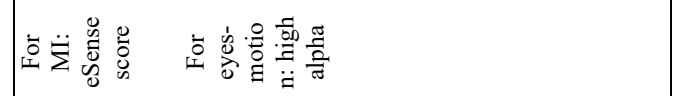 & $\frac{E}{\bar{z}} \overline{\mathrm{v}}$ \\
\hline 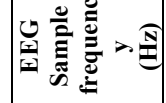 & i & $\frac{\pi}{n}$ & ஜ̆ \\
\hline 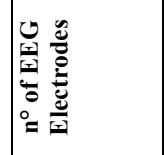 & 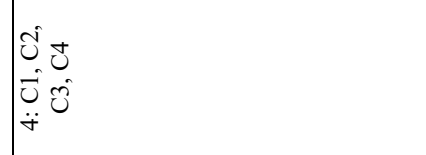 & $\stackrel{\overrightarrow{0}}{\stackrel{0}{*}}$ & 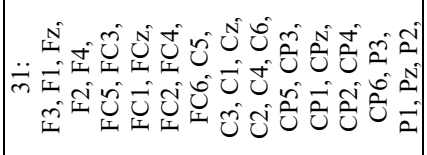 \\
\hline 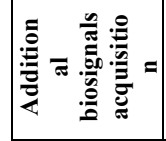 & 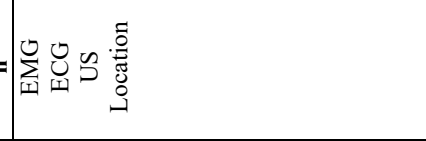 & z & そ \\
\hline ט. & 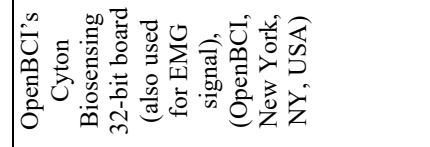 & 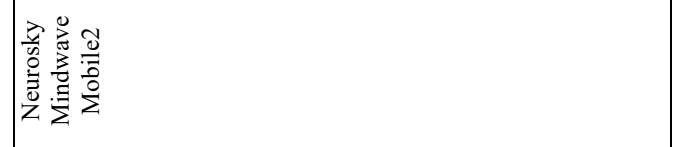 & 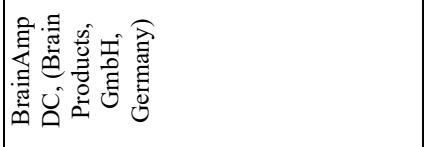 \\
\hline 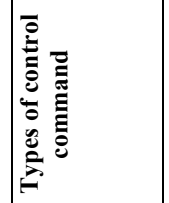 & 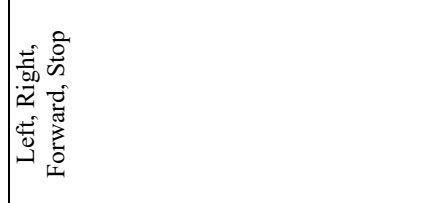 & 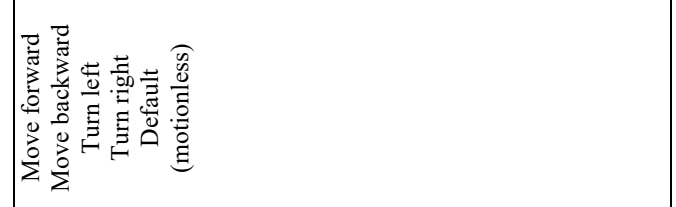 & 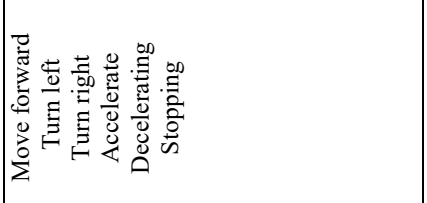 \\
\hline 詃 & 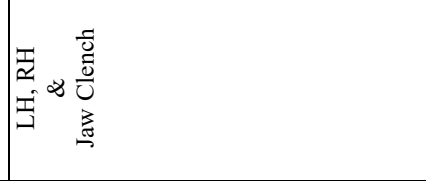 & 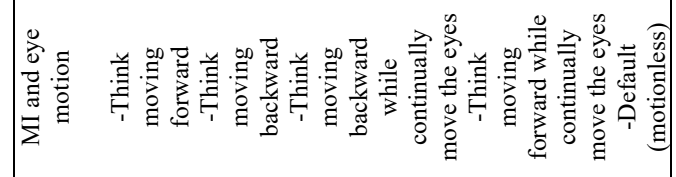 & 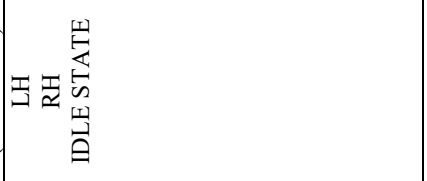 \\
\hline : & 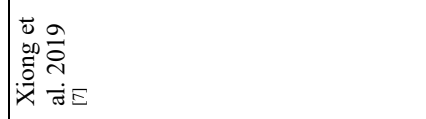 & 营 & 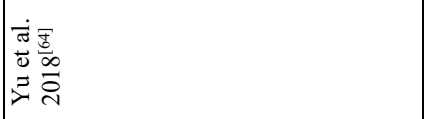 \\
\hline
\end{tabular}




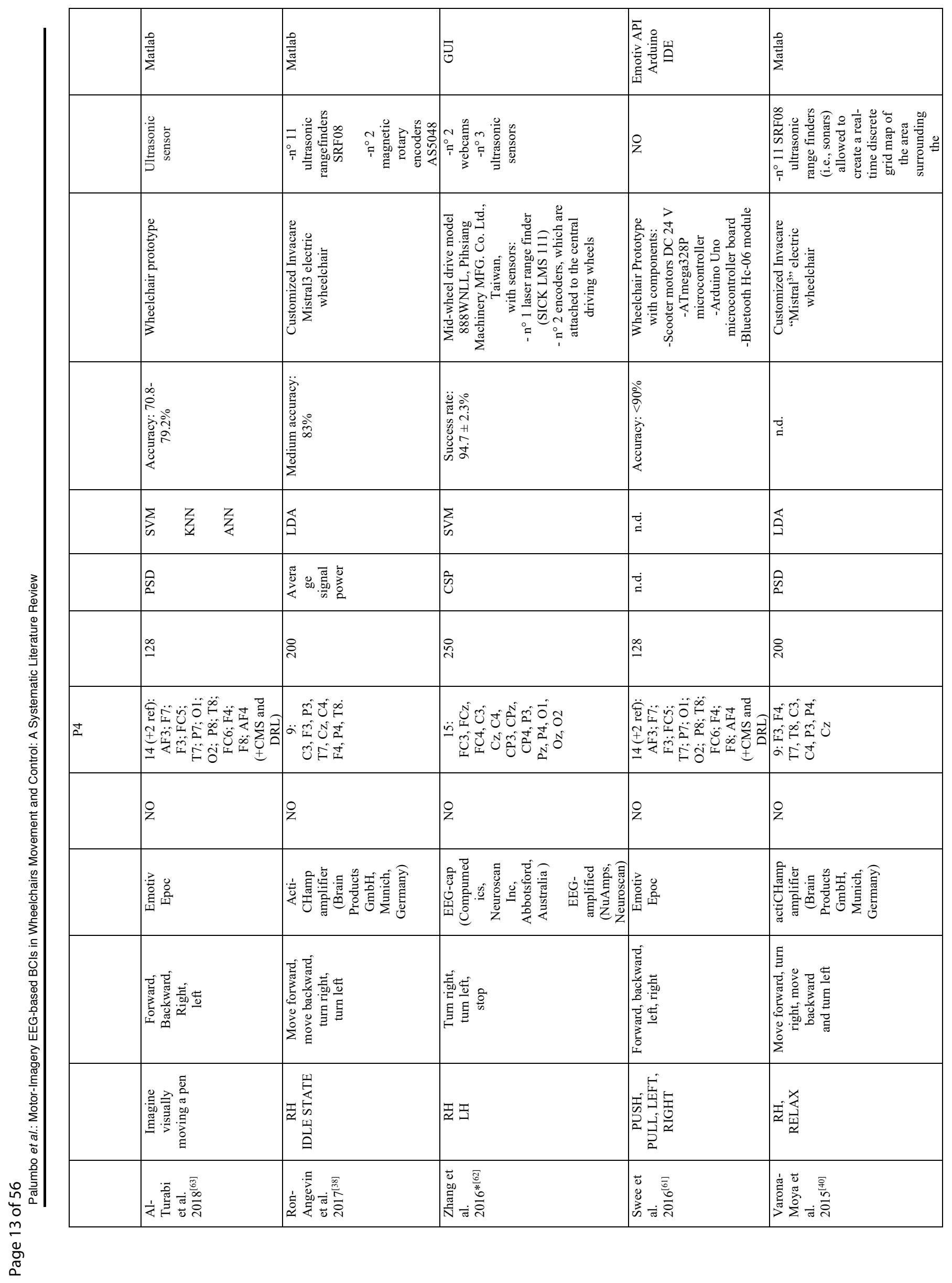




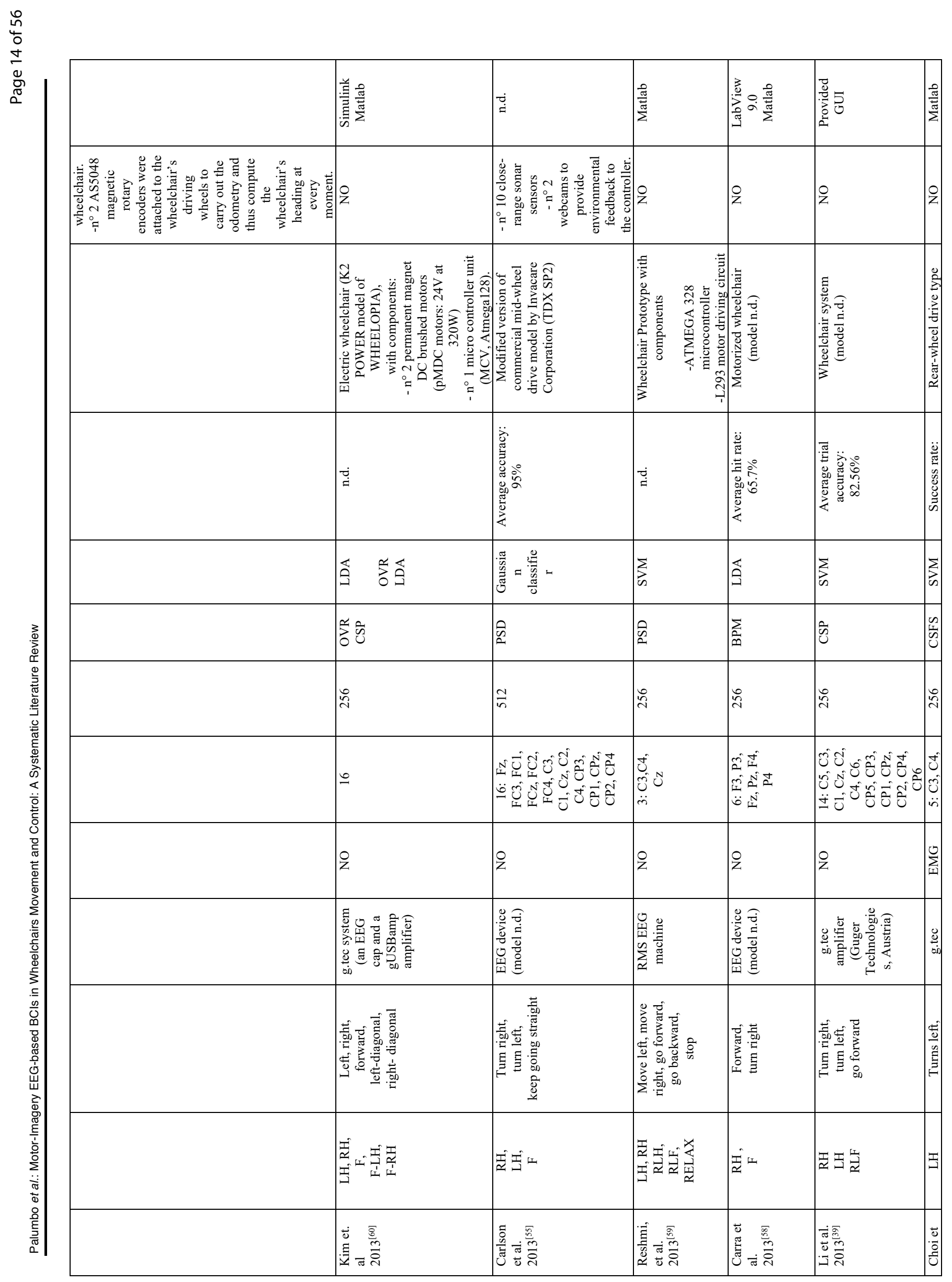




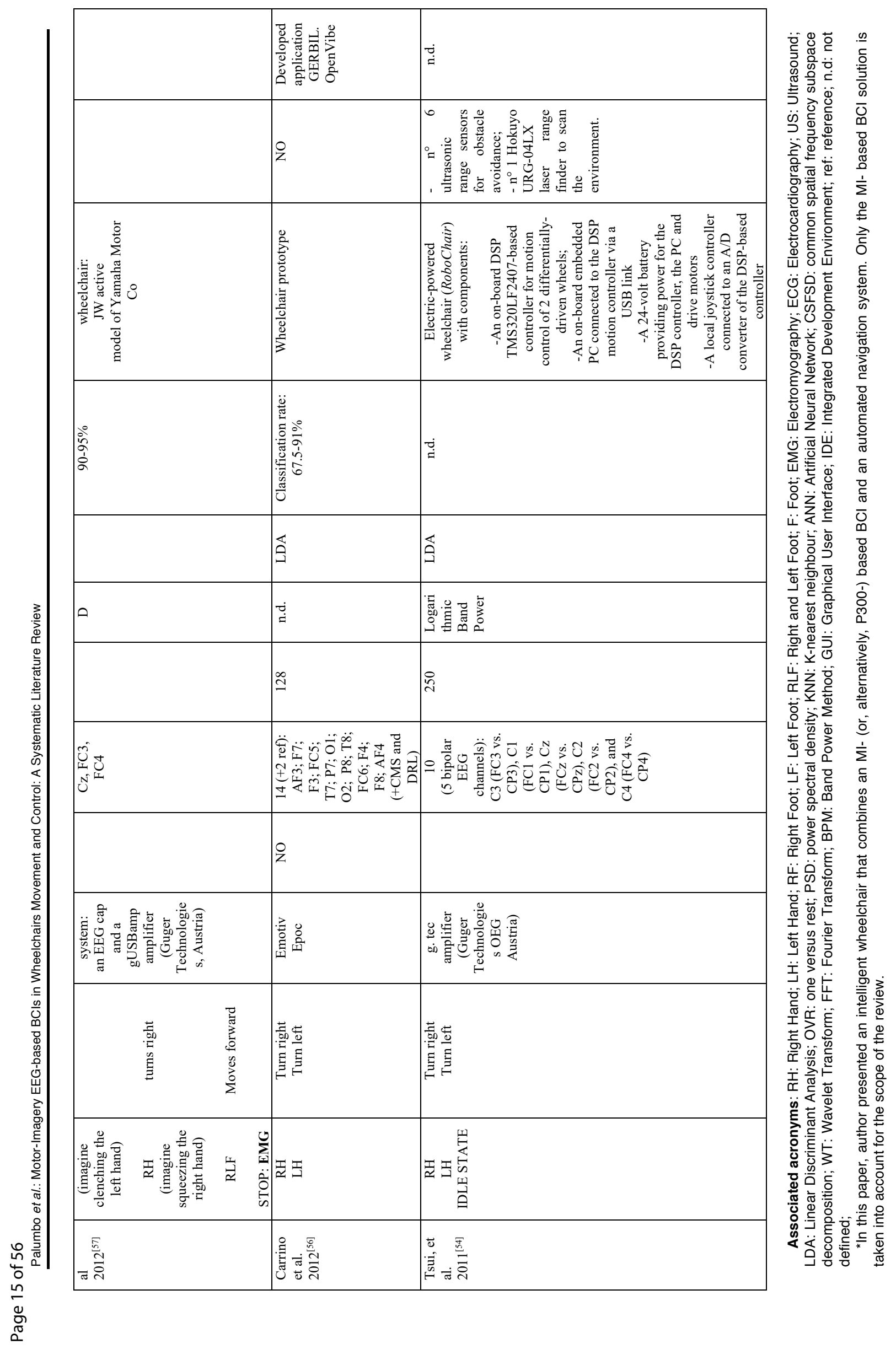




\section{REFERENCES}

[1] E. M. Smith et al., "Assistive Technology Use and Provision During COVID-19: Results From a Rapid Globa Survey," Int J Health Policy Manag, Nov 11 2020, doi: 10.34172/ijhpm.2020.210

[2] F. De Marchi et al., "Telehealth approach for amyotrophic lateral sclerosis patients: the experience during COVID-19 pandemic," Acta Neurol Scand, vol. 143, no. 5, pp. 489496, May 2021, doi: 10.1111/ane.13373.

[3] F. De Marchi, E. Contaldi, L. Magistrelli, R. Cantello, C Comi, and L. Mazzini, "Telehealth in Neurodegenerative Diseases: Opportunities and Challenges for Patients and Physicians," Brain Sci, vol. 11, no. 2, Feb 13 2021, doi: 10.3390/brainsci11020237.

[4] A. Palumbo, et al., "Cloud-based biomedical system for remote monitoring of ALS patients," in IEEE Int Conf Bioinformatics Biomed (BIBM), Seoul, Korea (South), 2020 pp. 1469-1476. doi: 10.1109/BIBM49941.2020.9313485.

[5] I. Lazarou, S. Nikolopoulos, P. C. Petrantonakis, I. Kompatsiaris, and M. Tsolaki, "EEG-Based BrainComputer Interfaces for Communication and Rehabilitation of People with Motor Impairment: A Novel Approach of the 21 (st) Century," Front Hum Neurosci, vol. 12, p. 14, 2018, doi: 10.3389/fnhum.2018.00014.

[6] T. M. Vaughan, "Brain-computer interfaces for people with amyotrophic lateral sclerosis," Handb Clin Neurol, vol. 168 pp. 33-38, 2020, doi: 10.1016/B978-0-444-63934-9.000044.

[7] M. Xiong et al., "A Low-Cost, Semi-Autonomous Wheelchair Controlled by Motor Imagery and Jaw Muscle Activation," in IEEE Int Conf Syst Man Cybern (SMC), Bari, Italy, 2019, pp. 2180-2185, DOI: 10.1109/SMC.2019.8914544.

[8] L. Bi, X. Fan and Y. Liu, "EEG-Based Brain-Controlled Mobile Robots: A Survey," in IEEE Trans Hum Mach Syst, vol. 43, no. 2, pp. 161-176, March 2013, doi: 10.1109/TSMCC.2012.2219046.

[9] N. M. Krishnan, M. Mariappan, K. Muthukaruppan, M. H A. Hijazi, and W. W. Kitt, "Electroencephalography (EEG) Based Control in Assistive Mobile Robots: A Review," in IOP Conf. Ser. Mater. Sci. Eng., vol. 121, p. 012017 2016/03 2016, doi: 10.1088/1757-899x/121/1/012017.

[10] A. Fernandez-Rodriguez, F. Velasco-Alvarez, and R. RonAngevin, "Review of real brain-controlled wheelchairs," $J$ Neural Eng, vol. 13, no. 6, p. 061001, Dec 2016, doi: 10.1088/1741-2560/13/6/061001.

[11] Z. T. Al-Qaysi, B. B. Zaidan, A. A. Zaidan, and M. S. Suzani, "A review of disability EEG based wheelchai control system: Coherent taxonomy, open challenges and recommendations," Comput Methods Programs Biomed, vol. 164, pp. 221-237, Oct 2018, doi 10.1016/j.cmpb.2018.06.012

[12] J. Leaman and H. M. La, "A Comprehensive Review of Smart Wheelchairs: Past, Present, and Future," in IEEE Trans Hum Mach Syst, vol. 47, no. 4, pp. 486-499, Aug. 2017, DOI: 10.1109/THMS.2017.2706727.

[13] M. Aljalal, S. Ibrahim, R. Djemal, and W. Ko "Comprehensive review on brain-controlled mobile robots and robotic arms based on electroencephalography signals," Intelligent Service Robotics, vol. 13, no. 4, pp. 539-563, 2020/10/01 2020, doi: 10.1007/s11370-020-00328-5.

[14] D. Moher, A. Liberati, J. Tetzlaff, D. G. Altman, and P. Group, "Preferred reporting items for systematic reviews and meta-analyses: the PRISMA statement," $J$ Clin Epidemiol, vol. 62, no. 10, pp. 1006-12, Oct 2009, doi: 10.1016/j.jclinepi.2009.06.005.

[15] A. Dev, M. A. Rahman and N. Mamun, "Design of an EEGBased Brain Controlled Wheelchair for Quadriplegic Patients," in 3rd International Conference for Convergence in Technology (I2CT), Pune, India, 2018, pp. 1-5, DOI: 10.1109/I2CT.2018.8529751.
[16] L. Xin, S. Gao, J. Tang and X. Xu, "Design of a Brain Controlled Wheelchair," in IEEE 4th International Conference on Control Science and Systems Engineering (ICCSSE), Wuhan, China, 2018, pp. 112-116, DOI: 10.1109/CCSSE.2018.8724794

[17] R. H. Abiyev, N. Akkaya, E. Aytac, I. Gunsel, and A. Cagman, "Brain-Computer Interface for Control of Wheelchair Using Fuzzy Neural Networks," Biomed Res Int, vol. 2016, p. 9359868, 2016, doi: $10.1155 / 2016 / 9359868$

[18] Q. Huang, Z. Zhang, T. Yu, S. He, and Y. Li, "An EEG/EOG-Based Hybrid Brain-Computer Interface: Application on Controlling an Integrated Wheelchair Robotic Arm System," Front Neurosci, vol. 13, p. 1243, 2019, doi: 10.3389/fnins.2019.01243.

[19] Y. Yu et al., "Self-Paced Operation of a Wheelchair Based on a Hybrid Brain-Computer Interface Combining Motor Imagery and P300 Potential," IEEE Trans Neural Syst Rehabil Eng, vol. 25, no. 12, pp. 2516-2526, Dec 2017, doi: 10.1109/TNSRE.2017.2766365.

[20] J. Long, Y. Li, H. Wang, T. Yu, J. Pan, and F. Li, "A hybrid brain computer interface to control the direction and speed of a simulated or real wheelchair," IEEE Trans Neural Syst Rehabil Eng, vol. 20, no. 5, pp. 720-9, Sep 2012, doi: 10.1109/TNSRE.2012.2197221.

[21] M. Rashid et al., "Current Status, Challenges, and Possible Solutions of EEG-Based Brain-Computer Interface: A Comprehensive Review," Front Neurorobot, vol. 14, p. 25 , 2020, doi: 10.3389/fnbot.2020.00025.

[22] N. Padfield, J. Zabalza, H. Zhao, V. Masero, and J. Ren, "EEG-Based Brain-Computer Interfaces Using MotorImagery: Techniques and Challenges," Sensors (Basel), vol. 19, no. 6, Mar 22 2019, doi: 10.3390/s19061423.

[23] D. Steyrl, R.J. Kobler, and G.R. Müller-Putz, "On Similarities and Differences of Invasive and Non-invasive Electrical Brain Signals in Brain-Computer Interfacing," $J$ Biomed Sci Eng, vol. 9, no. 8, pp. 393-398, Jul. 2016.

[24] C. H. Han, Y. W. Kim, D. Y. Kim, S. H. Kim, Z. Nenadic, and C. H. Im, "Electroencephalography-based endogenous brain-computer interface for online communication with a completely locked-in patient," J Neuroeng Rehabil, vol. 16, no. 1, p. 18, Jan 30 2019, doi: 10.1186/s12984-019-0493-0.

[25] R. Abiri, S. Borhani, E. W. Sellers, Y. Jiang, and X. Zhao, "A comprehensive review of EEG-based brain-computer interface paradigms," J Neural Eng, vol. 16, no. 1, p. 011001, Feb 2019, doi: 10.1088/1741-2552/aaf12e.

[26] G. R. Muller-Putz, R. Scherer, C. Brauneis, and G. Pfurtscheller, "Steady-state visual evoked potential (SSVEP)-based communication: impact of harmonic frequency components," J Neural Eng, vol. 2, no. 4, pp. 123-30, Dec 2005, doi: 10.1088/1741-2560/2/4/008.

[27] C. Han, G. Xu, J. Xie, C. Chen, and S. Zhang, "Highly Interactive Brain-Computer Interface Based on Flicker-Free Steady-State Motion Visual Evoked Potential," Sci Rep, vol. 8, no. 1, p. 5835, Apr 11 2018, doi: 10.1038/s41598-01824008-8.

[28] E. W. Sellers, D. J. Krusienski, D. J. McFarland, T. M. Vaughan, and J. R. Wolpaw, "A P300 event-related potential brain-computer interface $(\mathrm{BCI})$ : the effects of matrix size and inter stimulus interval on performance," Biol Psychol, vol. 73, no. 3, pp. 242-52, Oct 2006, doi: 10.1016/j.biopsycho.2006.04.007.

[29] M. Xu, X. Xiao, Y. Wang, H. Qi, T. P. Jung, and D. Ming, "A Brain-Computer Interface Based on Miniature-EventRelated Potentials Induced by Very Small Lateral Visual Stimuli," IEEE Trans Biomed Eng, vol. 65, no. 5, pp. 11661175, May 2018, doi: 10.1109/TBME.2018.2799661

[30] G. Onose et al., "On the feasibility of using motor imagery EEG-based brain-computer interface in chronic tetraplegics for assistive robotic arm control: a clinical test and longterm post-trial follow-up," Spinal Cord, vol. 50, no. 8, pp. 599-608, Aug 2012, doi: 10.1038/sc.2012.14

[31] J. Shin, J. Kwon, and C. H. Im, "A Ternary Hybrid EEGNIRS Brain-Computer Interface for the Classification of 
Brain Activation Patterns during Mental Arithmetic, Motor Imagery, and Idle State," Front Neuroinform, vol. 12, p. 5 2018, doi: 10.3389/fninf.2018.00005

[32] J. R. Wolpaw, N. Birbaumer, D. J. McFarland, G. Pfurtscheller, and T. M. Vaughan, "Brain-computer interfaces for communication and control," Clin Neurophysiol, vol. 113, no. 6, pp. 767-91, Jun 2002, doi: 10.1016/s1388-2457(02)00057-3.

[33] H. Serby, E. Yom-Tov, and G. F. Inbar, "An improved P300-based brain-computer interface," IEEE Trans Neural Syst Rehabil Eng, vol. 13, no. 1, pp. 89-98, Mar 2005, doi: 10.1109/TNSRE.2004.841878

[34] M. Middendorf, G. McMillan, G. Calhoun, and K. S. Jones, "Brain-computer interfaces based on the steady-state visualevoked response," IEEE Trans Rehabil Eng, vol. 8, no. 2 , pp. 211-4, Jun 2000, doi: 10.1109/86.847819.

[35] Y. Wang, R. Wang, X. Gao, B. Hong, and S. Gao, "A practical VEP-based brain-computer interface," IEEE Trans Neural Syst Rehabil Eng, vol. 14, no. 2, pp. 234-9, Jun 2006, doi: 10.1109/TNSRE.2006.875576.

[36] R. Millan Jdel, F. Renkens, J. Mourino, and W. Gerstner, "Noninvasive brain-actuated control of a mobile robot by human EEG," IEEE Trans Biomed Eng, vol. 51, no. 6, pp. 1026-33, Jun 2004, doi: 10.1109/TBME.2004.827086.

[37] K. Tanaka, K. Matsunaga and H. O. Wang, "Electroencephalogram-based control of an electric wheelchair," in IEEE Transactions on Robotics, vol. 21, no 4, pp. 762-766, Aug. 2005, doi: 10.1109/TRO.2004.842350

[38] R. Ron-Angevin, F Velasco-Alvarez, A FernandezRodriguez, A. Diaz-Estrella, M. J. Blanca-Mena, and F. J. Vizcaino-Martin, "Brain-Computer Interface application: auditory serial interface to control a two-class motorimagery-based wheelchair," J Neuroeng Rehabil, vol. 14 no. 1, p. 49, May 30 2017, doi: 10.1186/s12984-017-0261y.

[39] J. Li, J. Liang, Q. Zhao, J. Li, K. Hong, and L. Zhang, "Design of assistive wheelchair system directly steered by human thoughts," Int J Neural Syst, vol. 23, no. 3, p. 1350013, Jun 2013, doi: 10.1142/S0129065713500135.

[40] S. Varona-Moya, F. Velasco-Álvarez, S. Sancha-Ros, Á. Fernández-Rodríguez, M. J. Blanca and R. Ron-Angevin, "Wheelchair navigation with an audio-cued, two-class motor imagery-based brain-computer interface system," in 7th Int IEEE EMBS Conf Neural Eng (NER), Montpellier, France, 2015, pp. 174-177, doi: 10.1109/NER.2015.7146588.

[41] J. Tang, Y. Liu, D. Hu, and Z. Zhou, "Towards BCIactuated smart wheelchair system," Biomed Eng Online, vol. 17, no. 1, p. 111, Aug 20 2018, doi: 10.1186/s12938018-0545-x

[42] B. Rebsamen et al., "Controlling a wheelchair using a BCI with low information transfer rate," in IEEE 10th Int Conf Rehabil Robot, Noordwijk, Netherlands, 2007, pp. 10031008, doi: 10.1109/ICORR.2007.4428546.

[43] I. Iturrate, J. M. Antelis, A. Kubler and J. Minguez, "A Noninvasive Brain-Actuated Wheelchair Based on a P300 Neurophysiological Protocol and Automated Navigation," in IEEE Trans. Robot., vol. 25, no. 3, pp. 614-627, June 2009, doi: 10.1109/TRO.2009.2020347.

[44] R. Alqasemi and R. Dubey, "A 9-DoF Wheelchair-Mounted Robotic Arm System: Design, Control, Brain-Computer Interfacing, and Testing," in Advances in Robot Manipulators, Ernest Hall (Ed.), 2010, ISBN: 978-953-307070-4, InTech, Available from: http://www.intechopen .com/books/advances-inrobotmanipulators/a-9-dof-wheelchair-mounted-roboticarm-system-design-control-brain-computer-interfacingandtesting

[45] B. Shin, T. Kim and S. Jo, "Non-invasive brain signal interface for a wheelchair navigation," in ICCAS Gyeonggi-do, Korea (South), 2010, pp. 2257-2260, doi: 10.1109/ICCAS.2010.5669830.

[46] A. C. Lopes, G. Pires, and U. Nunes, "Assisted navigation for a brain-actuated intelligent wheelchair," Robot Auton
Syst, vol. 61, no. 3, pp. 245-258, 2013/03/01/ 2013, doi https://doi.org/10.1016/j.robot.2012.11.002.

[47] C. Mandel, T. Lüth, T. Laue, T. Röfer, A. Gräser and B. Krieg-Brückner, "Navigating a smart wheelchair with a brain-computer interface interpreting steady-state visual evoked potentials," in IEEE/RSJ International Conference on Intelligent Robots and Systems, St. Louis, MO, USA, 2009, pp. 1118-1125, doi: 10.1109/IROS.2009.5354534

[48] Z. Xu, J. Li, R. Gu and B. Xia, "Steady-State Visually Evoked Potential (SSVEP)-Based Brain-Computer Interface (BCI): A Low-Delayed Asynchronous Wheelchair Control System," in: Huang T., Zeng Z., Li C., Leung C.S. (eds) Neural Information Processing. ICONIP 2012. Lecture Notes in Computer Science, vol 7663. Springer, Berlin, Heidelberg. https://doi.org/10.1007/978-3-642-34475-6_37

[49] S.M.T. Müller, T.F. Bastos and M.S. Filho, "Proposal of a SSVEP-BCI to Command a Robotic Wheelchair", J Control Autom Electr Syst, vol. 24, pp. 97-105, 2013. https://doi.org/10.1007/s40313-013-0002-9

[50] J. Duan, Z. Li, C. Yang and P. Xu, "Shared control of a brain-actuated intelligent wheelchair," in Proceeding of the 11th World Congress on Intelligent Control and Automation, Shenyang, China, 2014, pp. 341-346, doi: 10.1109/WCICA.2014.7052737.

[51] D. W. Ng, Y. Soh and S. Goh, "Development of an Autonomous BCI Wheelchair," in IEEE Symp. Comput. Intell. Brain Comput. Interfaces (CIBCI), Orlando, FL, USA, 2014, pp. 1-4, doi: 10.1109/CIBCI.2014.7007784.

[52] Y. Li, J. Pan, F. Wang, and Z. Yu, "A hybrid BCI system combining P300 and SSVEP and its application to wheelchair control," IEEE Trans Biomed Eng, vol. 60, no. 11, pp. 3156-66, Nov 2013, doi: 10.1109/TBME.2013.2270283

[53] L. Cao, J. Li, H. Ji, and C. Jiang, "A hybrid brain computer interface system based on the neurophysiological protocol and brain-actuated switch for wheelchair control," J Neurosci Methods, vol. 229, pp. 33-43, May 30 2014, doi 10.1016/j.jneumeth.2014.03.011.

[54] C. S. Tsui, J. Q. Gan, and H. Hu, "A self-paced motor imagery based brain-computer interface for robotic wheelchair control," Clin EEG Neurosci, vol. 42, no. 4, pp. 225-9, Oct 2011, doi: 10.1177/155005941104200407.

[55] T. Carlson and J. del R. Millan, "Brain-Controlled Wheelchairs: A Robotic Architecture," in IEEE Robot Autom Mag, vol. 20, no. 1, pp. 65-73, March 2013, doi: 10.1109/MRA.2012.2229936.

[56] F. Carrino, J. Dumoulin, E. Mugellini, O. A. Khaled and R. Ingold, "A self-paced BCI system to control an electric wheelchair: Evaluation of a commercial, low-cost EEG device," in ISSNIP Biosignals and Biorobotics Conference: Biosignals and Robotics for Better and Safer Living (BRC), Manaus, Brazil, 2012, pp. 1-6, DOI 10.1109/BRC.2012.6222185

[57] K. Choi, "Control of a vehicle with EEG signals in real-time and system evaluation," Eur J Appl Physiol, vol. 112, no. 2, pp. 755-66, Feb 2012, doi: 10.1007/s00421-011-2029-6.

[58] M. Carra and A. Balbinot, "Evaluation of sensorimotor hythms to control a wheelchair," in ISSNIP Biosignals and Biorobotics Conference: Biosignals and Robotics for Better and Safer Living (BRC), Rio de Janeiro, Brazil, 2013, pp. 14, DOI: 10.1109/BRC.2013.6487456.

[59] G. Reshmi and A. Amal, "Design of a BCI System for Piloting a Wheelchair Using Five Class MI Based EEG," in Third International Conference on Advances in Computing and Communications, Cochin, India, 2013, pp. 25-28, doi: 10.1109/ICACC.2013.12

[60] K. Kim, T. Carlson and S. Lee, "Design of a robotic wheelchair with a motor imagery-based brain-computer interface," in International Winter Workshop on BrainComputer Interface (BCI), Gangwon, Korea (South), 2013, pp. 46-48, DOI: 10.1109/IWW-BCI.2013.6506625.

[61] S.K. Swee, K.D.T. Kiang and L.Z. You, "EEG Controlled Wheelchair," Proc. MATEC Web Conf., Apr. 2016. Available: https://doi.org/10.1051/matecconf/20165102011. 
[62] R. Zhang et al., "Control of a Wheelchair in an Indoor Environment Based on a Brain-Computer Interface and Automated Navigation," IEEE Trans Neural Syst Rehabil Eng, vol. 24, no. 1, pp. 128-39, Jan 2016, doi: 10.1109/TNSRE.2015.2439298.

[63] H. Al-Turabi and H. Al-Junaid, "Brain-computer interface for wheelchair control in smart environment," Smart Cities Symposium 2018, Bahrain, 2018, pp. 1-6, DOI: 10.1049/cp.2018.1391.

[64] Y. Yu, Y. Liu, J. Jiang, E. Yin, Z. Zhou, and D. Hu, "An Asynchronous Control Paradigm Based on Sequential Motor Imagery and Its Application in Wheelchair Navigation," IEEE Trans Neural Syst Rehabil Eng, vol. 26, no. 12 , pp. 2367-2375, Dec 2018, doi: 10.1109/TNSRE.2018.2881215.

[65] K. Permana, S. K. Wijaya, and P. Prajitno, "Controlled wheelchair based on brain computer interface using Neurosky Mindwave Mobile 2," in AIP Conference Proceedings, vol. 2168, no. 1, p. 020022, 2019, doi: $10.1063 / 1.5132449$.

[66] M. Soufineyestani, D. Dowling, and A. Khan, "Electroencephalography (EEG) Technology Application and Available Devices," vol. 10, no, 21, p. 7453, 2020. [Online]. Available: https://www.mdpi.com/2076$3417 / 10 / 21 / 7453$.

[67] X. Gu et al., "EEG-based Brain-Computer Interfaces (BCIs): A Survey of Recent Studies on Signal Sensing Technologies and Computational Intelligence Approaches and Their Applications," IEEE/ACM Trans Comput Bio Bioinform, vol. PP, Jan 19 2021, doi: 10.1109/TCBB.2021.3052811.

[68] Available: https://www.emotiv.com/epoc/

[69] N. S. Williams, G. M. McArthur, and N. A. Badcock, "10 years of EPOC: A scoping review of Emotiv's portable EEG device," J BioRxiv, p. 2020.07.14.202085, 2020, doi: 10.1101/2020.07.14.202085\%

[70] Available:https://www.gtec.at/product-configurator/gusbamp/

[71] Available:https://www.brainproducts.com/products by app s.php?aid=5

[72] X. Jiang, G.-B. Bian, and Z. Tian, "Removal of Artifacts from EEG Signals: A Review," vol. 19, no. 5, p. 987, 2019. [Online]. Available: https://www.mdpi.com/1424 $8220 / 19 / 5 / 987$.

[73] S. Aggarwal and N. Chugh, "Signal processing techniques for motor imagery brain computer interface: A review," Array, vol. 1-2, p. 100003, 2019/01/01/ 2019, doi https://doi.org/10.1016/j.array.2019.100003.

[74] A. Singh, A. A. Hussain, S. Lal, and H. W. Guesgen, "A Comprehensive Review on Critical Issues and Possible Solutions of Motor Imagery Based Electroencephalography Brain-Computer Interface," Sensors (Basel), vol. 21, no. 6, Mar 20 2021, doi: 10.3390/s21062173.

[75] H. Ramoser, J. Muller-Gerking and G. Pfurtscheller, "Optimal spatial filtering of single trial EEG during imagined hand movement," in IEEE Trans. Rehab. Eng., vol. 8, no. 4, pp. 441-446, Dec. 2000, doi: $10.1109 / 86.895946$

[76] F. Lotte, M. Congedo, A. Lecuyer, F. Lamarche, and B. Arnaldi, "A review of classification algorithms for EEGbased brain-computer interfaces," J Neural Eng, vol. 4, no. 2, pp. R1-R13, Jun 2007, doi: 10.1088/1741-2560/4/2/R01.

[77] V. N. Vapnik, The nature of statistical learning theory. New York: Springer, 1995, pp. xv, 188 p.

[78] A. Tyagi and V. Nehra, "Classification of motor imagery EEG signals using SVM, k-NN and ANN," CSI Transactions on ICT, vol. 4, no. 2, pp. 135-139, 2016/12/01 2016, doi: 10.1007/s40012-016-0091-2.

[79] Available: http://openvibe.inria.fr/

[80] Available:https://docs.openbci.com/docs/06Software/01Ope nBCISoftware/GUIDocs

[81] Y. Matanga, K. Djouani and K. Anish, "A Matlab/Simulink framework for real time implementation of endogenous brain computer interfaces," 2017 IEEE AFRICON, Cape
Town, South Africa, 2017, pp. 100-105, doi 10.1109/AFRCON.2017.8095463.

[82] B Venthur, S. Dahne, J. Hohne, $\mathrm{H}$. Heller, and B. Blankertz, "Wyrm: A Brain-Computer Interface Toolbox in Python," Neuroinformatics, vol. 13, no. 4, pp. 471-86, Oct 2015, doi: 10.1007/s12021-015-9271-8.

[83] Available: https://github.com/bbci/wyrm

[84] G. Schalk, D. J. McFarland, T. Hinterberger, N. Birbaumer, and J. R. Wolpaw, "BCI2000: a general-purpose braincomputer interface (BCI) system," IEEE Trans Biomed Eng, vol. 51, no. 6, pp. 1034-43, Jun 2004, doi: 10.1109/TBME.2004.827072.

[85] Available:https://www.bci2000.org/mediawiki/index.php/M ain Page

[86] Z. Tayeb et al., "Gumpy: a Python toolbox suitable for hybrid brain-computer interfaces," J Neural Eng, vol. 15, no. 6, p. 065003, Dec 2018, doi: 10.1088/17412552/aae186.

[87] C. A. Kothe and S. Makeig, "BCILAB: a platform for braincomputer interface development," J Neural Eng, vol. 10, no. 5, p. 056014 , Oct 2013, doi: 10.1088/17412560/10/5/056014.

[88] Available: https://www.nitrc.org/projects/bcilab/

[89] Available: http://gumpy.org/

[90] Y. LeCun, Y. Bengio, and G. Hinton, "Deep learning," Nature, vol. 521, no. 7553, pp. 436-44, May 28 2015, doi: 10.1038 /nature 14539.

[91] S. Hochreiter and J. Schmidhuber, "Long short-term memory," Neural Comput, vol. 9, no. 8, pp. 1735-80, Nov 15 1997, doi: 10.1162/neco.1997.9.8.1735.

[92] A. Palumbo, P. Vizza, P. Veltri, A. Gambardella, F. Pucci and M. Sturniolo, "Design of an electronic device for braincomputer interface applications," 2009 IEEE International Workshop on Medical Measurements and Applications, Cetraro, Italy, 2009, pp. 99-103, DOI: 10.1109/MEMEA.2009.5167963.

[93] A. Palumbo et al., "An Embedded System for EEG Acquisition and Processing for Brain Computer Interface Applications," in Wearable and Autonomous Biomedical Devices and Systems for Smart Environment: Issues and Characterization, A. Lay-Ekuakille and S. C. Mukhopadhyay Eds. Berlin, Heidelberg: Springer Berlin Heidelberg, 2010, pp. 137-154.

[94] F. Schettini, F. Aloise, M. Mecella, M. Caruso, A. Palumbo, A. Pizzimenti, M. Inghilleri and F. Cincotti. "From Keyboard to Brain-Computer Interface: the Brindisys Project", in GNB2012, Rome, Italy, 2012. ISBN: 97888 $5553182-5$.

[95] F. Schettini, A. Riccio, L. Simione, G. Liberati, M. Caruso, B. Calabrese, N. Ielpo, A. Palumbo, V. Frasca, M. Mecella, F. Amato, A. Pizzimenti, M. Inghilleri, and F. Cincotti. " The Brindisys Project: Brain-computer interface devices to support individual autonomy in locked-in individuals", in $3^{\circ}$ Convegno Arisla: Nuove Prospettive di ricerca per un futuro senza SLA, Milano, Italia, 2012.

[96] F. Schettini et al., "The Brindisys project: Brain Computer Interfaces as assistive technology for people with ALS", Fifth International Brain-Computer Interface Meeting, Pacific Grove, California, 2013. In: Proceedings of the Fifth International Brain-Computer Interface Meeting: Defining the Future, 2013, doi:10.3217/978-3-85125-260-6-186.

[97] Available: https://arrigopalumbo.com/pubblicazioni/

[98] Available: https://biomedical.arrigopalumbo.com/progettosimple/

[99] Available: http://www.alessioforlano.com 


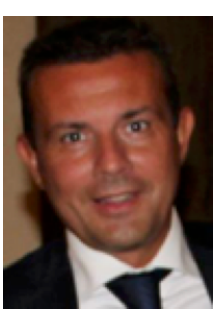

A. Palumbo received a Master's Degree in Computer Science Engineering (major in Electronics and Telecommunications) at the University of Calabria and then he received a $\mathrm{PhD}$ in Electronics Engineering at the University "Mediterranea" of Reggio Calabria. He is currently contract professor at the University "Magna Graecia" of Catanzaro (Italy). During his career he has taught "Analog and Digital Electronics devices" and some other courses at the Faculty of Computer Science and Biomedical Engineering (University "Magna Graecia" of Catanzaro). His research activities are concerned with innovative embedded systems (FPGA and DSP) in order to develop both industrial applications and high performances electronics devices, aiming to digital signals processing in biomedical environment. Some of the research projects have been published in International Journals, Conferences and Books.

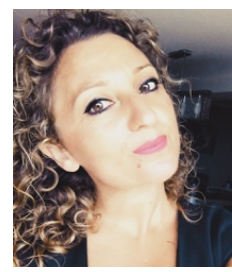

v. Gramigna was born in Cetraro (CS), Italy in 1980. In 2007 she received her master degree in Mechanical Engineering at the University of Calabria. In 2011 she obtained a PhD in Biomedical and Informatic Engineering at the "Magna Graecia" University of Catanzaro. During post-graduate period she continued entertaining interests on biorobotics at "Biorobotic Institute"Scuola Superiore Sant'Anna in Pisa. In the 2014, at the Neuroscience Research Center of the "Magna Graecia" University of Catanzaro, scientific interest has veered strongly towards the cognitive neuroscience. Her main research concentration is application of several neuroimaging methodologies (fNIRS, fMRI, EEG, TMS) to the study of neuronal activity and hemodynamic processes in neurodegenerative diseases.

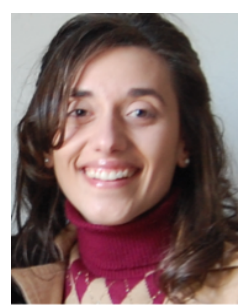

B. Calabrese received her master's degree with honors in Electronic Engineering at the University of Calabria in the year 2008 and a Ph.D. in Biomedical and Informatic Engineering at the University of "Magna Graecia" of Catanzaro in 2012. She was a research fellow at the University of "Magna Graecia" from 2012 to 2019. Currently, with Dr. Palumbo Arrigo, Ph.D., and Dr. Nicola lelpo, Ph.D., she has been granted a research grant for the SIMPLE (Smart Solutions for health monitoring and independent mobility for elderly and disabled people) project by the Italian Ministry of Education, University and Research (MIUR). Her main research interests include brain-computer interface design, biomedical signal processing, and acquisition. The results of her research activities include numerous international publications.

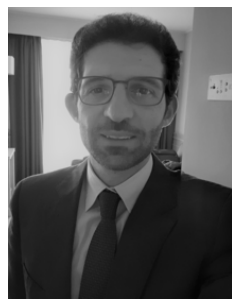

N. lelpo was born in Maratea (PZ), Italy in 1983. $\mathrm{He}$ received master's degree in Electronic Engineering at the University of Calabria in 2008 and a PhD in Biomedical and Informatic Engineering at the "Magna Graecia" University of Catanzaro in 2013. His research interest include Brain Computer Interface, biomedical signal acquisition and biomedical electronic circuits design. He was a contract professor at the University of Calabria end at the "Magna Graecia" University of Catanzaro from 2014 to today. 